\title{
Escamas y huesos de pescado en la dieta sobre el desempeño zootécnico de codornices japonesas en fase de postura
}

\section{Flakes and bones fish in the diet on performance zootechnical of Japanese}

\author{
quail in posture phase
}

\author{
Hurtado Nery Victor Libardo¹, Gómez Beltrán David Alberto²y \\ Herrera Yineth Maryori² \\ ${ }^{1}$ Médico Veterinario Zootecnista, MSc., PhD, Docente Universidad de los Llanos y \\ ${ }^{2}$ Médicos Veterinarios Zootecnistas Universidad de los Llanos \\ vhurtado@unillanos.edu.co
}

Recibido 24 de Enero 2017, Aceptado 21 de Abril 2017

\section{RESUMEN}

Con el objetivo de evaluar el efecto de la inclusión de escamas y huesos de pescado como fuentes de calcio y fósforo, sobre el desempeño zootécnico de codornices japonesas en fase de postura. Se utilizaron 200 aves de 70 días de edad, durante 26 semanas, las cuales fueron distribuidas en un diseño experimental completamente al azar con cuatro tratamientos, cinco repeticiones y 10 aves cada una. Los tratamientos consistieron en incluir escamas y huesos de pescado en los siguientes niveles: $0 ; 0.54 ; 1.08$ y $1.62 \%$. La producción de huevos fue de: 84.3, 85.6, 86.1 y 79.5\%; consumo diario: 25.4, 25.6, 25.2 y $24.3 \mathrm{~g}$; conversión alimenticia: $0.398,0.401,0.409$ y $0.392 \mathrm{~kg}$ de ración/docena de huevos; masa de huevo: 2.90. 2.84, 2.66 y 2.89 para los niveles con 0. 0.54, 1.08 y $1.62 \%$, respectivamente. Los resultados indican incremento cuadrático $(P<0.05)$, según la ecuación $Y=-7.9401 x^{2}+9.4237 x+83.96, R^{2}=0.897$, por efecto de la escama y huesos de pescado sobre la producción y mejoramiento de la masa de huevos por kg de ración. En conclusión, estas dos fuentes de calcio y fósforo se pueden incluir en la dieta para codornices en fase de postura hasta en $0.59 \%$ porque mejoran la producción de huevos y la conversión alimenticia dada en kg de ración/kg de huevo, sin afectar las otras variables de desempeño. 
Palabras clave: Coturnix japonica, producción de huevos, minerales, reciclaje de residuos.

\begin{abstract}
In order to evaluate the effect of the inclusion of scales and fish bones as sources of calcium and phosphorus, on the zootechnical performance of Japanese quail stance phase. 200 birds of 70 days of age were used for 26 weeks, which were distributed in a completely randomized experimental design with four treatments, five replicates and 10 birds each. The treatments consisted of including fish scales and bones at the following levels: $0 ; 0.54 ; 1.08$ y $1.62 \%$. Egg production was: 84.3 , 85.6, 86.1 and 79.5\%; daily consumption: 25.4, 25.6, 25.2 and $24.3 \mathrm{~g}$; feed conversion: $0.398,0.401,0.409$ and $0.392 \mathrm{~kg}$ of ration/dozen eggs; egg mass: 2.90. 2.84, 2.66 and 2.89; for levels with $0.0 .54,1.08$ and $1.62 \%$, respectively. The results indicate quadratic increase $(P<0.05)$, according to equation: $Y=-7.9401 x^{2}$ $+9.4237 x+83.96, R^{2}=0.897$, by effect of fish scale and bones on the production and improvement of egg mass per $\mathrm{kg}$ of ration. In conclusion, these two sources of calcium and phosphorus can be included in the diet for quail in the posture phase up to $0.59 \%$ because they improve egg production and the feed conversion given in $\mathrm{kg}$ of ration $/ \mathrm{kg}$ of egg, without affecting the other performance variables.
\end{abstract}

Keywords: Coturnix japonica, egg production, minerals, waste recycling.

\title{
RESUMO
}

Com o fim de avaliar o efeito da inclusão de escalas e ossos de peixes como fontes de cálcio e de fósforo, sobre o desempenho zootécnico de codornas japonesas en fase de postura. 200 aves de 70 dias de idade foram usados durante 26 semanas, que eles foram distribuídos em um desenho experimental totalmente ao acaso, com quatro tratamentos, cinco repetições e 10 aves cada uma. Os tratamentos consistiam em incluir escalas e ossos de peixes nos seguintes níveis: $0 ; 0.54 ; 1.08$ y $1.62 \%$. A produção de ovos foi: $84.3,85.6,86.1$ e $79.5 \%$; consumo diário: 25.4, 25.6, 25.2 e 24.3 g; conversão alimentar: 0398, 0401, 0409 e 0392 kg de ração/dúzia de ovos; massa de ovos: 2.90. 2.84, 2.66 e 2.89; para os níveis 0 . 
0.54, 1.08 e $1.62 \%$, respectivamente. Os resultados indicam aumento quadrático $(P<0.05)$ de acordo com a equação: $Y=-7.9401 x^{2}+9.4237 x+83.96, R^{2}=0.897$, o efeito de escamas de peixe e ossos sobre a produção e melhoramento da massa de ovos por $\mathrm{kg}$ de ração. Em conclusão, estas duas fontes de cálcio e fósforo podem ser incluídos na dieta de codornas em fase de posição até $0.59 \%$ porque melhoram a produção de ovos e conversão alimentar dada em $\mathrm{kg}$ ração $/ \mathrm{kg}$ de ovo, sem afetar outras variáveis de desempenho.

Palavras-chave: Coturnix japonica, produção de ovos, minerais, reciclagem de resíduos.

\section{INTRODUCCIÓN}

La acuicultura es un renglón de la economía que presenta buen ritmo de crecimiento y rentabilidad superiores a otras actividades agropecuarias (FAO, 2016) la cual también genera problemas ambientales como el desvío de las trayectorias de fuentes hídricas, contaminación del agua con desecho de escamas e incluso vísceras, puesto que los residuos no se aprovechan en procesos productivos. La escama de Piaractus brachypomus y Oreochromis sp, según Gómez y Benítez, (2011) contiene 40\% carbonato de calcio y $10 \%$ de fósforo total, lo que posibilita su utilización en dietas con altos requerimientos de estos minerales, como es el caso de las codornices en fase de postura. Las escamas de pescado también son utilizadas en la producción de artesanías (Costa et al., 2016).

Los residuos de la piscicultura también son utilizados en forma de ensilaje biológicos para la alimentación animal, en forma de harina de pescado contiendo residuos de carne, piel, cabeza, espinas, cartílagos (Abreu et al., 2012). Estos residuos de pescado son ricos en compuestos orgánicos, generando impactos ambientales negativos (Feltes et al., 2010), los restos óseos tienen potencial de ser utilizados como fuente de calcio y fosforo, lo cual favorece a la industria avícola porque se reducen los costos de alimentación (Godoy et al., 2016). 
La cría de codornices se constituye en una alternativa para la alimentación del ser humano, la cual puede ser utilizada para producir carne o huevos, en ambos propósitos la dieta debe contener las fuentes nutrientes como fósforo y calcio necesarios para óptimo desempeño zootécnico (Silva et al 2009; 2011) y obtener carne o el huevo de excelente calidad (Costa et al., 2010).

Según Barreto et al., (2007) del total de calcio en el organismo 98 a 99\% está en los huesos y el resto participa del metabolismo celular, este mineral es esencial para la calidad de la cáscara del huevo. Entre tanto, el fósforo es el mineral que más encarece la ración, siendo indispensable para el ave porque participa de la formación del esqueleto y también contribuye en la formación de la cáscara del huevo, se ha comprobado que el calcio se encuentra en la harina de huesos y las espinas del pescado (FEDNA, 2016; Costa et al., 2011).

Con base en lo expuesto anteriormente, el objetivo de este trabajo fue evaluar el efecto de la inclusión de escamas y huesos de pescado procesados en un sistema de flujo continuo, sobre el desempeño zootécnico de codornices japonesas en fase de postura.

\section{METODOLOGÍA}

El trabajo fue realizado en la Universidad de los Llanos, Villavicencio, Colombia (latitud $4^{\circ} 04^{\prime} 30.93^{\prime \prime} \mathrm{N}$, longitud $73^{\circ} 34^{\prime} 55.78$ " O), a una altitud de $423 \mathrm{msnm}$, temperatura promedio de $27^{\circ} \mathrm{C}$, precipitación de $4050 \mathrm{~mm}$, humedad relativa del $80 \%$ correspondiente a la zona de vida de bosque muy húmedo tropical (bmh-T) (Silva et al., 2013).

La fase experimental tuvo una duración de 26 semanas, en la cual las aves fueron alojadas en galpón cubierto, con ventilación natural, en jaulas metálicas dotadas de comederos lineales y bebederos automáticos, las dietas y agua fueron suministradas a voluntad, las codornices recibían 14 horas de luz diarias natural y artificial, las heces y residuos de alimento fueron sometidos a compostaje. Los huevos se recolectaron en la mañana, se pesaron en balanza digital y se colocaron en empaque plástico para su conservación y distribución. 
Se utilizaron 200 codornices de 70 días de edad, distribuidas en un diseño experimental completamente al azar, con cuatro tratamientos, cinco repeticiones y 10 aves en cada una, los datos fueron sometidos a análisis de variancia y de regresión polinomial en el programa Sistema de Analises Estatísticas e Genéticas, (SAEG, 2007). Los tratamientos fueron isoproteicos (20\%) e isoenegéticos (2887 kcal de energía metabolizable) y se establecieron de acuerdo a la inclusión de huesos y escamas de pescado en los siguientes niveles: $0 ; 0.54 ; 1.08$ y $1.62 \%$ en la dieta. Como proceso de adecuación, los huesos y escamas fueron sometidas a calor continuo durante 3 horas para remover impurezas, posteriormente secado y molido para ser incluido en las raciones experimentales (Tabla 1), las cuales fueron formuladas para atender los requerimientos nutricionales para codornices japonesas recomendados por National Research Council (1994), El análisis de composición nutricional de los ingredientes y las dietas fue realizado en el Laboratorio de Nutrición Animal de UNILLANOS (Tabla 2).

Tabla 1. Composición centesimal de raciones con cuatro niveles de escama y huesos de pescado para codornices japonesas en postura

\begin{tabular}{ccccc}
\hline \multirow{2}{*}{ Materia prima } & \multicolumn{4}{c}{ Niveles de escama de pescado } \\
\cline { 2 - 5 } & $\mathbf{0 \%}$ & $\mathbf{0 . 5 4 \%}$ & $\mathbf{1 . 0 8 \%}$ & $\mathbf{1 . 6 2 \%}$ \\
\hline Maíz & 56.4 & 56.4 & 56.4 & 56.4 \\
Torta de soja & 33.9 & 33.9 & 33.9 & 33.9 \\
Aceite vegetal & 1.45 & 1.45 & 1.45 & 1.45 \\
Carbonato de Ca & 5.41 & 5.02 & 4.65 & 4.27 \\
Escama y huesos & 0.00 & 0.54 & 1.08 & 1.62 \\
Fosfato bicálcico & 1.32 & 1.04 & 0.74 & 0.45 \\
Sal & 0.50 & 0.50 & 0.50 & 0.50 \\
Vitamina $^{1}$ & 0.30 & 0.30 & 0.30 & 0.30 \\
D- L metionina & & 0.17 & 0.17 & 0.17 \\
L-lisina & 0.17 & 0.05 & 0.05 & 0.05 \\
Material inerte $^{4}$ & 0.05 & 0.68 & 0.80 & 0.94 \\
Costo por Kilo $\$$ & 0.55 & 1499.43 & 1461.73 & 1425.16 \\
(Pesos Colombianos) $^{\text {Pes }}$ & 1534.87 & & & \\
\hline
\end{tabular}

${ }^{1}$ Composición por kg de producto. Vitamina A 7500000 IU; vitamina D 1500 IU; vitamina E 7 IU; Menadiona $1250 \mathrm{mg}$; vitamina B1 $500 \mathrm{mg}$; vitamina B2 $5000 \mathrm{mg}$; niacina $35000 \mathrm{mg}$; ácido pantoténico $10000 \mathrm{mg}$; vitamina B12 $2000 \mathrm{mg}$; ácido $1000 \mathrm{mg}$; biotina $50 \mathrm{mg}$. ${ }^{2} \mathrm{DL}$ metionina 99\%. ${ }^{3}$ L-Lisina $78 \% .{ }^{4}$ Arenilla 
Tabla 2. Composición Nutricional de las dietas para codornices de postura

\begin{tabular}{ccccc}
\hline \multirow{2}{*}{ Nutriente } & \multicolumn{4}{c}{ Niveles de escama de pescado } \\
\cline { 2 - 5 } & $\mathbf{0 \%}$ & $\mathbf{0 . 5 4 \%}$ & $\mathbf{1 . 0 8 \%}$ & $\mathbf{1 . 6 2 \%}$ \\
\hline Proteína bruta, \% & 20.0 & 20.0 & 20.0 & 20.0 \\
EM kcal/kg* & 2887 & 2887 & 2887 & 2887 \\
P disponible, \%* & 0.35 & 0.35 & 0.35 & 0.35 \\
Calcio, \%* & 2.50 & 2.50 & 2.50 & 2.50 \\
Lisina, \%* & 1.00 & 1.00 & 1.00 & 1.00 \\
Metionina, \%* & 0.45 & 0.45 & 0.45 & 0.45 \\
Treonina, \%* & 0.67 & 0.67 & 0.67 & 0.67 \\
Fibra bruta, \% & 2.82 & 2.82 & 2.82 & 2.82 \\
\hline
\end{tabular}

* Valores Calculados. Promedio de tres muestras, Laboratorio de Nutrición Animal UNILLANOS

\section{RESULTADOS Y DISCUSIÓN}

Los niveles de inclusión de la harina escamas y huesos de pescado en la dieta para codornices japonesas aumentan $(P<0.05)$ en forma cuadrática $\left(y=-7.9401 x^{2}\right.$ $\left.+9.4237 x+83.96, R^{2}=0.897\right)$ la postura de huevos y mejora la cantidad de ración por $\mathrm{kg}$ de masa de huevo $(\mathrm{P}<0.05)$, el desarrollo de la ecuación de regresión permite estimar en $0.59 \%$ (Tabla 3 y Grafica1) el nivel de inclusión de harina escama y de huesos de pescado para máxima producción de huevos.

Tabla 3. Desempeño zootécnico de codornices japonesas en postura con diferentes niveles de escamas y huesos de pescado

\begin{tabular}{|c|c|c|c|c|c|c|}
\hline \multirow{2}{*}{ Variable } & \multicolumn{6}{|c|}{ Nivel inclusión de escamas y huesos de pescado, \% } \\
\hline & 0 & 0.54 & 1.08 & 1.62 & (p) & CV \\
\hline Días experimentales & 180 & 180 & 180 & 180 & & \\
\hline Consumo Total, g & 4572 & 4608 & 4536 & 4374 & & \\
\hline Postura de huevos ${ }^{1}, \%$ & 84.3 & 85.7 & 86.1 & 79.5 & 0.05 & 4.85 \\
\hline Consumo diario de ración, $g$ & 25.4 & 25.6 & 25.2 & 24.3 & 0.17 & 3.68 \\
\hline $\begin{array}{l}\text { Conversión alimenticia, kg } \\
\text { de ración/docena de huevos }\end{array}$ & 0.40 & 0.40 & 0.41 & 0.39 & 0.58 & 4.90 \\
\hline $\begin{array}{l}\text { Conversión alimenticia }{ }^{2}, \mathrm{~kg} \\
\text { de ración/kg de huevos }\end{array}$ & $2.90^{a}$ & $2.84^{a b}$ & $2.66^{b}$ & $2.89^{a}$ & 0.05 & 5.05 \\
\hline
\end{tabular}




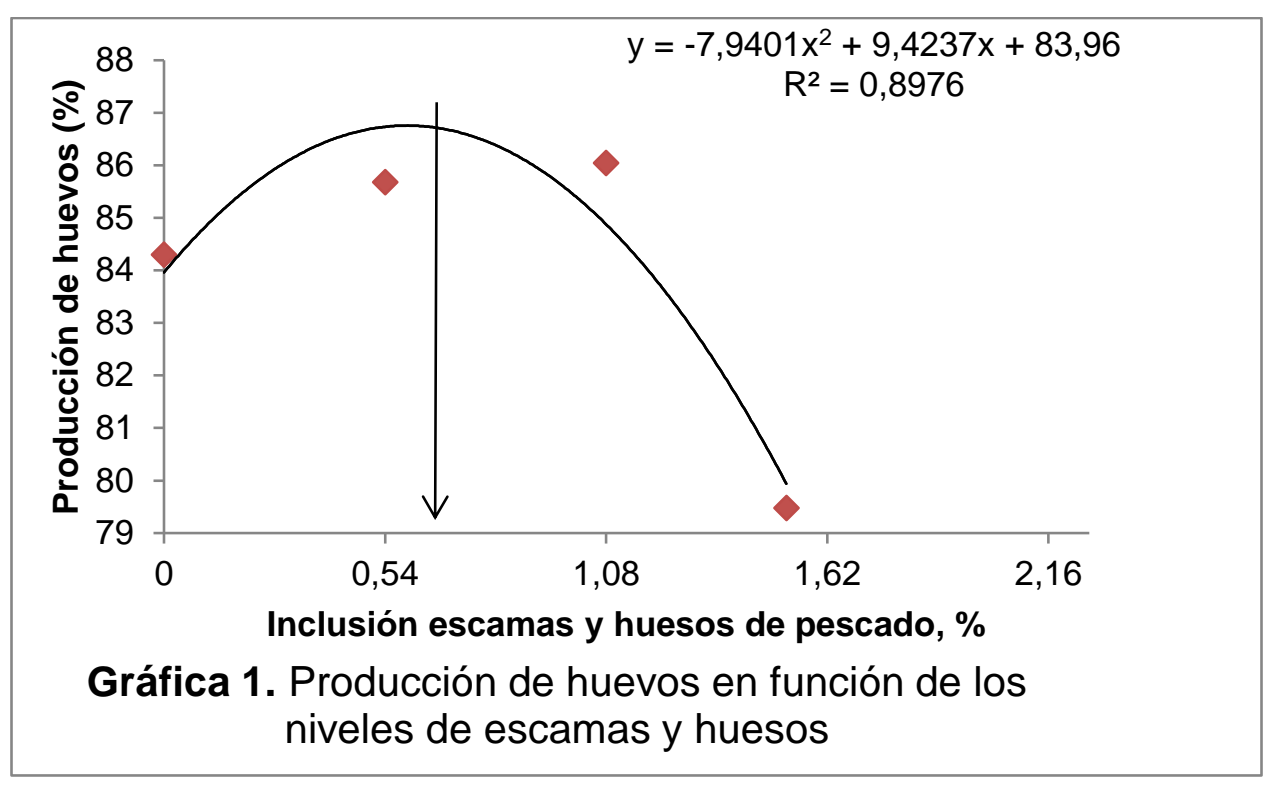

Los niveles de inclusión de la harina escamas y huesos de pescado en la dieta para codornices japonesas aumentan la postura y mejora la cantidad de ración por $\mathrm{kg}$ de masa de huevo $(\mathrm{P}<0.05)$, se estimó en $0.59 \%$ (Gráfica 1 ) el nivel de inclusión de harina escama y huesos de pescado para máxima producción de huevos, aun sin estimar la solubilidad de cada uno de los minerales en estudio incorporados en la ración; mientras que las otras variables no fueron influenciadas por la sustitución del carbonato de calcio por la harina escama y huesos de pescado.

Los resultados pueden sugerir que las aves toleran otros ingredientes de los descritos en NRC, (1994) y Rostagno et al., (2011), como fuentes de calcio y fósforo, sin afectar el desempeño zootécnico de codornices japonesas en fase de postura. Del mismo modo, la utilización como alimento para aves de un residuo con posible impacto ambiental, que en su deposición final puede contaminar las fuentes de agua, permite que las escamas y huesos de pescado, mediante procesos sencillos se conviertan en una fuente no convencional de fósforo y calcio, que puede sustituir el fosfato bicálcico en $0.21 .2,43.9$ y $65.9 \%$ como fuente de fosforo y en $0.7 .21,14.121 .1 \%$ al carbonato de calcio como fuente de este mineral, en la dieta para codornices en postura. 
Los resultados obtenidos superan la producción de huevos constatada por Melo et al., (2008) utilizando fosfato monoamónico y harina de algas marinas como fuentes alternativas de minerales; en contraste, los resultados de producción de huevos fueron menores a los obtenidos por Reis et al., (2012) con la inclusión de $3.44 \%$ de harina de cáscara de huevo en la dieta. Los niveles de harina.

Las fuentes energéticas como maíz y aceite vegetal, y proteínicas se mantuvieron constantes en los cuatro tratamientos (Tabla 1), por lo tanto la variabilidad en el aprovechamiento de estos dos nutrientes para la producción de huevos estuvo influenciada por las fuentes de $\mathrm{Ca}$ y $\mathrm{P}$, tanto es así que el consumo y la producción de huevos se redujeron cuando se incluyó 1.08\% de escamas y harina de huesos (Figuras 2 y 3), lógicamente que al disminuir el consumo, también afecta el aprovechamiento de la proteína y energía y por tanto la producción y calidad de los huevos.

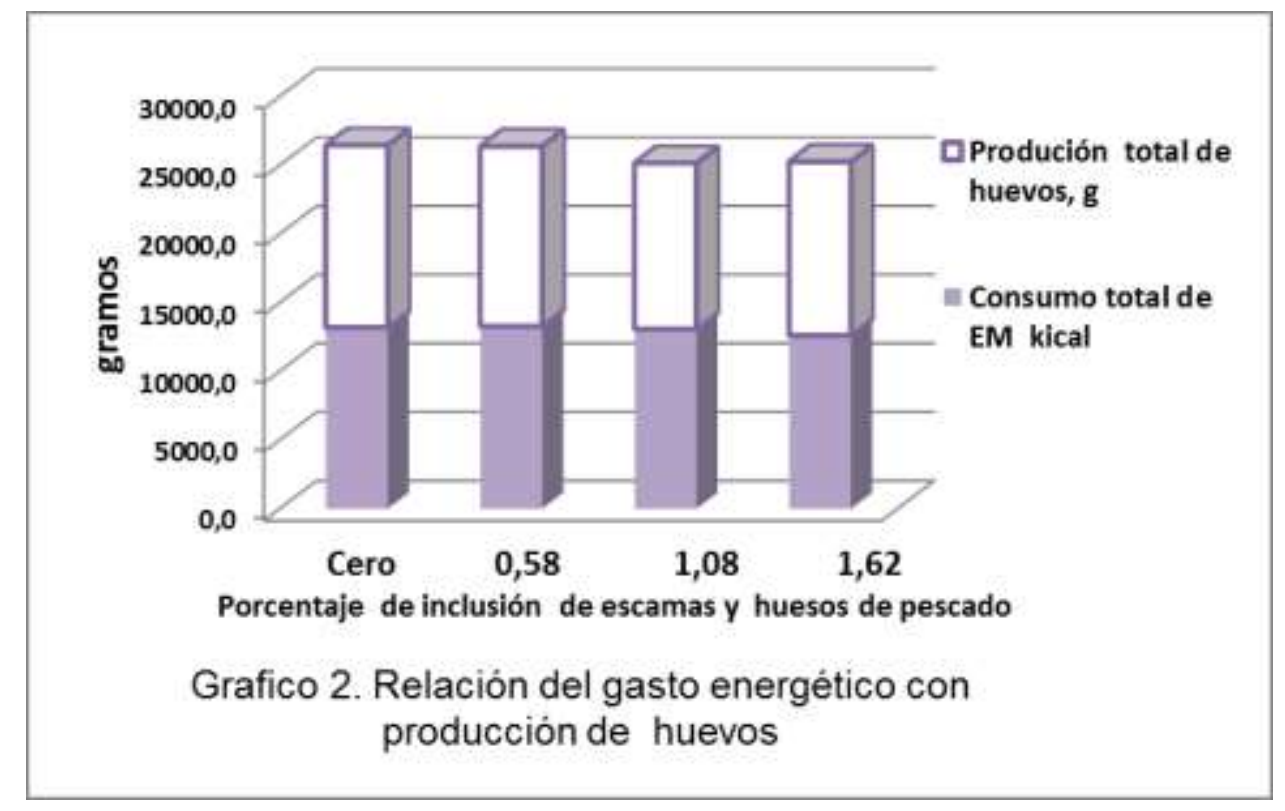

Es de anotar, que a medida que se aumenta el nivel de reemplazo de las escamas y huesos de pescado en la dieta de las codornices el costo por kilo disminuyó de manera ascendente en 2,30. 5,0 y 7,16\% en comparación con el cero por ciento de inclusión (Tabla 1), no hay que desconocer que las fuentes tradicionales de fosforo y calcio son más costosas en comparación con estos residuos de la piscicultura, que al rescatarlos y someterlos a un tratamiento de flujo continuo se 
pueden incluir en las dietas como fuentes de calcio y 'fosforo, siendo este último importante en procesos metabólicos para el aprovechamiento energético, factor que mantiene la producción de huevo evitando la pérdida de peso.

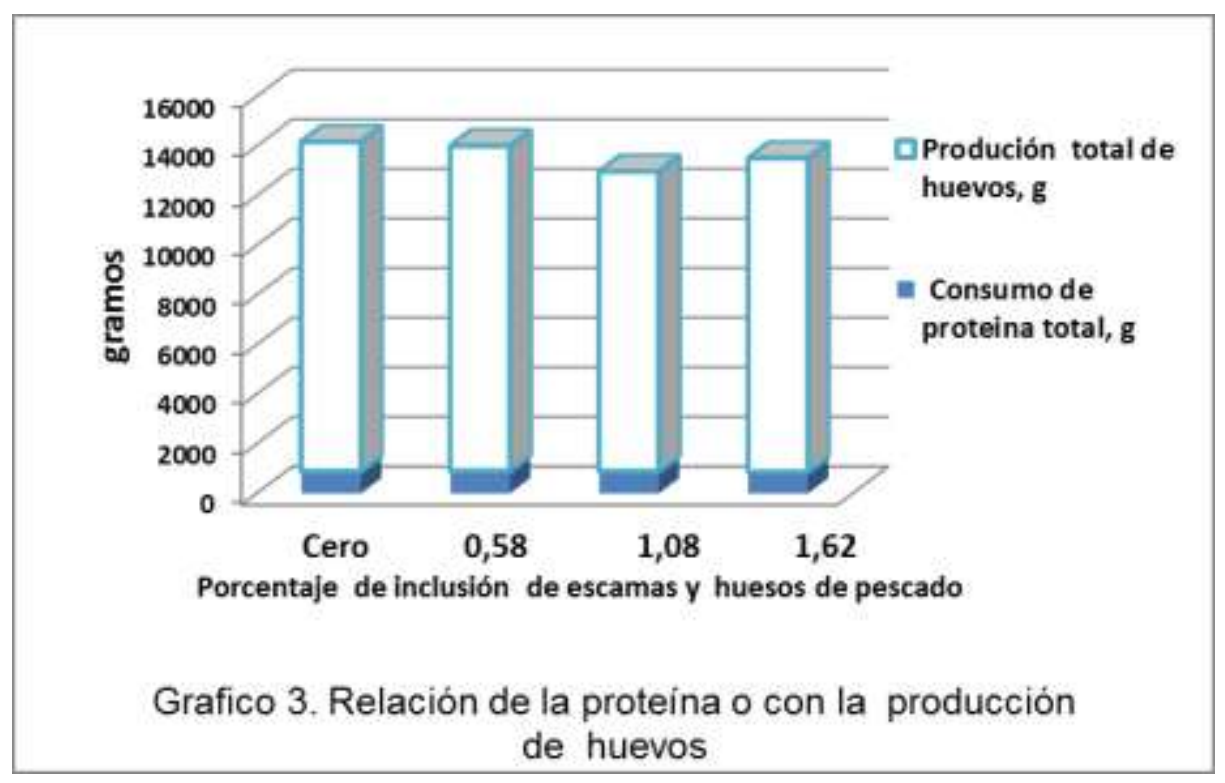

\section{CONCLUSIONES}

Se sugiere incluir hasta $0.59 \%$ de harina de huesos y escamas de pescado como fuente de fosforo y calcio en la dieta para codornices en postura entre las 10 y 36 semanas de edad para incrementar la producción de huevos, aprovechando un desecho de la actividad piscícola, potencialmente contaminante de fuentes de agua.

\section{REFERENCIAS BIBLIOGRÁFICAS}

1. Abreu LF, Ribeiro SCA, Araujo EAF. Processo agroindustrial: elaboração de farinha de resíduos de tambaqui (Colossoma macroppomum) para uso como ingrediente de rações de pescado. Circular Técnica 47, Embrapa, 5 p. 2012.

2. Barreto SLT, Pereira CA, Umigi RT, da Rocha TC, Araujo MS, Silva CS, Torres Filho RA. Determinação da exigência nutricional de cálcio de codornas japonesas na fase inicial do ciclo de produção. Revista Brasileira de Zootecnia, 36 (1): 68-78. 2007.

3. Costa CHR, Barreto SLT, Umigi RT, Lima HJA, Araújo MS, Medina P. Balanço de cálcio e fósforo e estudo dos níveis desses minerais em dietas para codornas japonesas (45 a 57 semanas de idade), Revista Brasileira de Zootecnia, 39 (8):17481755. 2010.

4. Costa CHR, Barreto SLT, Gomes PC, Hosoda LH, Lipari CA, Lima HJA. Níveis de fósforo disponível em dietas para codornas japonesas de 45 a 57 semanas de idade. Revista Brasileira de Zootecnia, 40 (10): 2152-2160. 2011. 
5. Costa WM, Vidal JMA, Veiga MCM, Rodrigues JM, Santos JF. Aproveitamento de resíduos de pescado: o artesanato com escamas de peixe. Rev. Ciênc. Ext., 12 (2): 8-17, 2016.

6. FAO National Aquaculture Sector Overview. Visión general del sector acuícola nacional - Colombia. National Aquaculture Sector Overview Fact Sheets. Texto de Salazar Ariza, G. En: Departamento de Pesca y Acuicultura de la FAO. Roma. Recuperado 24 Septiembre de 2016. Disponible En: http://www.fao.org/fishery/countrysector/naso colombia/es

7. Feltes MMC, Corrêia JFG, Beirão LH, Block JM, Ninow JL, Spiller VR. Alternativas para a agregação de valor aos resíduos da industrialização de peixe. Revista Brasileira de Engenharia Agrícola e Ambiental, 14 (6): 669-677. 2010.

8. Fundación Española para el Desarrollo de la Nutrición Animal, FEDNA. Fuentes de calcio. 2016. Recuperado 16 Abril de 2016. Disponible En: http://www.fundacionfedna.org/ingredientes para piensos/fuentes-de-calcio

9. Godoy AC, Fries E, Corrêia AF, Melo IWA, Rodrigues RB, Boscolo WR. Apparent digestibility of fish meat and bone meal in Nile tilapia. Arch. Zootec. 65 (251): 341-348. 2016.

10. Gómez J, Benítez MJ. Evaluar el posible uso de la escama de cachama (Piaractus brachypomus) y tilapia (Oreochromis s.p.) como desecho de la producción piscícola en la producción de nutrición animal y como enmienda en suelos de la región. Tesis de grado Médico Veterinario Zootecnista. Villavicencio. Universidad de los Llanos. 33 p. 2011.

11. Melo TV, Ferreira RA, Carneiro JBA, Oliveira VC, Moura AMA, Silva CS, Nery, VLH. Performance of japanese quails utilizing seaweed flour and monoamonium phosphate Archivos de Zootecnia, 57 (219): 381-384. 2008.

12. National Research Council (NRC). Nutrient Requirements of Poultry: Ninth Revised Edition. National Academy Press Washington, D.C. 1994.

13. Reis RS, Barreto SLT, Lima HJA, Paula E, Muniz JCL, Mencalha R, Viana GS, Barbosa LMR. Substituição do calcário por farinha de casca de ovo na dieta de codornas japonesas no período de 40 a 52 semanas de idade. Revista Brasileira de Agropecuária Sustentável, 2 (1): 107-112. 2012.

14. Rostagno HS. Albino LFT, Donzele JL, Gomes PC, Oliveira RF, Lopes DC, Ferreira AS, Barreto SLT, Euclides RF. Tabelas Brasileiras para aves e suínos. Composição de alimentos e exigências nutricionais. 3a ed. Viçosa: UFV. 252 p. 2011.

15. Sistema para Análises Estatísticas (SAEG). Versão 9.1: Fundação Arthur Bernardes UFV - Viçosa. 2007.

16. Silva JD, Queiroz AC. Análise de alimentos Métodos químicos e biológicos. 3ª ed. Viçosa: UFV, 235 p. 2004.

17. Silva RM, Furlan AC, Ton APS, Martins EN, Scherer C, Murakami AE. Exigências nutricionais de cálcio e fósforo de codornas de corte em Crescimento. Revista Brasileira de. Zootecnia, 38 (8): 1509-1517. 2009.

18. Silva JHV, Jordão Filho J, Costa FGP, Lacerda PB, Vargas DGV. Nutritional requirements of quais. XXI Congresso Brasileiro de Zootecnia, Maceió. 15 p. 2011.

19. Silva AC, Zuluaga AM., Roa ML. Evaluación de la utilización de Cratylia argentea como suplemento en dietas para pollos de engorde. Rev. Sist. Prod. Agroecol, 4 (1): 140-152. 2013. 